LIVER

\title{
Angiopoietin 2 displays a vascular endothelial growth factor dependent synergistic effect in hepatocellular carcinoma development in mice
}

\author{
H Yoshiii, S Kuriyama, R Noguchi, J Yoshii, Y Ikenaka, K Yanase, T Namisaki, \\ M Kitade, M Uemura, T Masaki, H Fukui
}

See end of article for authors' affiliations

.....................

Correspondence to: Dr H Yoshiji, Third Department of Internal Medicine, Nara Medical University, Shijo-cho 840, Kashihara, Nara 6348521, Japan; yoshiijih@ naramed-u.ac.jp

Revised version received 28 June 2005

Accepted for publication 2 July 2005 Published online first 20 July 2005

Background: Orchestration of two major classes of angiogenic factors-namely, vascular endothelial growth factor (VEGF) and angiopoietin 2 (Ang-2)-has been shown to play a pivotal role in tumour angiogenesis, including hepatocellular carcinoma (HCC). However, few studies have focused on the direct interaction of these factors on in vivo tumour development and angiogenesis.

Aim: To examine the interaction between both factors in murine HCC.

Methods: We examined the combination effect of VEGF and Ang-2 overexpression by means of a combination of a retroviral tetracycline (tet) regulated gene manipulating system in vivo, by providing tet in the drinking water, and a conventional plasmid gene expression system.

Results: Neither Ang-2 nor VEGF overexpression induced proliferation of HCC cells in vitro. In vivo, although overexpression of Ang-2 did not increase tumour development, simultaneous expression of Ang2 and VEGF synergistically augmented tumour growth and angiogenesis in murine HCC. Ang-2 plus VEGF induced tumour development was markedly attenuated by treatment with neutralising monoclonal antibodies against VEGF receptors. Ang-2 plus VEGF overexpression significantly increased the activities of matrix metalloproteinase (MMP)-2 and MMP-9 in the tumour. Suppression of intratumoral VEGF almost completely abolished this augmentation of MMPs.

Conclusions: These results suggest that Ang-2 synergistically augments VEGF mediated HCC development and angiogenesis. This proangiogenic activity was exerted only in the presence of VEGF, at least partly mediated via induction of MMP-2 and MMP-9 in the tumour.

$\mathrm{H}$ epatocellular carcinoma (HCC) is one of the most prevalent malignancies worldwide and causes more than one million deaths annually. Its incidence continues to increase not only in Asia but also in the USA. ${ }^{12}$ One of the notable features of HCC in clinical practice is its hypervascularity but vascularity varies widely during the development of HCC. The type of vascularisation is not uniform, and abnormal blood flow can lead to dissemination of tumour cells in portal tracts. ${ }^{3}{ }^{4}$ However, the mechanism of neovascularisation during HCC development has still not been defined.

It is now widely recognised that angiogenesis plays a pivotal role in the development of solid tumours, including HCC. ${ }^{5}{ }^{6}$ It has been shown that two central endothelium specific growth factor families coordinate vascular development-namely, vascular endothelial growth factor (VEGF) and angiopoietins (Ang). ${ }^{78}$ Among the identified proangiogenic factors, VEGF is the most intriguing with regard to angiogenesis. ${ }^{7}$ In human specimens, increased expression of VEGF correlated with aggressive behaviour and poor prognosis. In animal experimental models, overexpression of VEGF enhanced tumour growth whereas suppression of VEGF inhibited tumour growth. ${ }^{7}{ }^{10}$ Two tyrosine kinases, fms-like tyrosine kinase (flt-1: VEGFR-1) and the kinase insert domain containing receptor/murine homologue, fetal liver kinase-1 (KDR/Flk-1: VEGFR-2), both of which are type III tyrosine kinase receptors, have been identified as the main VEGF receptors. VEGF expression was upregulated in the tumours of HCC compared with non-cancerous lesions, and that overexpression of VEGF exerted a marked increase in HCC development accompanied by augmentation of neovascularisation. ${ }^{11-13}$ We have reported that treatment with neutralising monoclonal antibodies (mAbs) against VEGFR-1 and VEGFR-2 (R-1mAb and R-2mAb, respectively) significantly suppressed murine HCC development associated with suppression of angiogenesis. ${ }^{14} 15$

Angiopoietins 1 and 2 (Ang-1, Ang-2) have been implicated in further remodelling of the initial microvasculature, and their biological actions are mediated through the Tie-2 receptor. ${ }^{16}{ }^{17}$ Unlike VEGF, neither Ang-1 nor Ang-2 produces a mitogenic signal via Tie-2 on endothelial cells (EC). It has been reported that Ang-1 promotes the stabilisation and tightening of the developing vessels whereas Ang-2 induces vascular destabilisation through blocking activation of Tie-2 by Ang-1. To date, conflicting results have been reported regarding the role of the Ang/Tie-2 system in tumour angiogenesis. These contradictory results pertain to overexpression of Ang-1 and Ang-2 in different tumours, suggesting a proangiogenic effect and an antiangiogenic effect for Ang-1 and Ang-2 in tumours, respectively. ${ }^{16}$ In HCC, it has been noted that Ang-1 was equally expressed in tumour and adjacent non-cancerous lesions whereas Ang-2 expression was significantly increased in the tumour compared with adjacent tissues. ${ }^{18}{ }^{19}$ Furthermore, nude mice

Abbreviations: $\alpha$-SMA, $\alpha$ smooth muscle actin; Ang-1, Ang-2, angiopoietin 1 and 2, respectively; APMA, p-aminophenylmercuric acetate; bFGF, basic fibroblast growth factor; BNL-HCC, BNL 1.7R hepatocellular carcinoma; EC, endothelial cell; ECM, extracellular matrix; ELISA, enzyme linked immunosorbent assay; HCC,

hepatocellular carcinoma; tet, tetracycline; mAb, monoclonal antibody; MMP, matrix metalloproteinase; MVD, microvessel density; PCR, polymerase chain reaction; VEGF, vascular endothelial growth factor; VEGFR-1, fms-like tyrosine kinase (flt-1); VEGFR-2, kinase-insert domain containing receptor/fetal liver kinase-1 (KDR/Flk-1) 
injected with a HCC cell line overexpressing Ang-2 showed faster tumour growth with greater neovascularisation in the tumour. ${ }^{18}$ These findings suggest that Ang-2 plays an important role in tumour development and angiogenesis in HCC.

It has also been reported that Ang-2 displays VEGF dependent modulation of angiogenesis. Ang-2 stimulated angiogenesis in the presence of VEGF whereas it caused vessel regression in the absence of VEGF in a corneal assay. ${ }^{20}{ }^{21} \mathrm{~A}$ recent report has shown the synergistic effect of Ang-2 and VEGF on induction of cardiac angiogenesis using a double transgenic mouse model..$^{22}$ The phenotype of the tumour EC is different from that of the normal EC at the molecular level. ${ }^{23}$ To date, our current knowledge on the coordination of VEGF and Ang-2 in tumour development and angiogenesis, including HCC, is very limited.

The Tet system is a tetracycline regulated gene expression system. ${ }^{24}$ This system allows manipulation of the gene of interest in an "on and off" manner in vivo whereas the conventional gene expression system cannot achieve either overexpression or suppression. In this study, we used a retrovirus mediated modified vector in which two components of the Tet systems were organised within the same vector in the opposite direction, resulting in a decrease in basal gene expression levels (Retro-Tet system). ${ }^{13}$

In the current study, we focused on orchestration of Ang-2 and VEGF in HCC development and angiogenesis by means of a combination of the Retro-Tet system and a conventional plasmid gene expression system in the murine HCC model. We also investigated the possible mechanism of orchestration of Ang-2 and VEGF in tumour development and angiogenesis.

\section{METHODS}

\section{Construction of the vector, and compounds}

A complete description of the construction of the Retro-Tet vector and Balb/c mice derived BNL.1ME A 7R. I (BNL) HCC cell line has been reported previously. ${ }^{13}$ In a preliminary study, we found that this BNL-HCC cell line expressed very low levels of endogenous Ang-2 and VEGF, and it did not express VEGFR-1, VEGFR-2, or Tie-2, as described previously. ${ }^{13}{ }^{14}$ Human Ang-2 cDNA was cloned into the multicloning site of the parent PBSTR-1 vector at a Bam Hl site to form the Tet-Ang-2 vector (fig 1A). For VEGF gene expression, we used a Pcl-neo plasmid vector (Promega, Forward, Wisconsin, USA). Human VEGF cDNA was cloned into the Eco Rl, Not 1 site of the Pcl-neo expression vector to form Pcl-neo-VEGF. As the Tet-Ang-2 vector and Pcl-neo expression vector were selected by different drugs (puromycin and geneticin, respectively), both expression systems could be introduced into the same cells. R-1mAb and R$2 \mathrm{mAb}$ were generated as described previously ${ }^{14} 25-27$ and the specific neutralisation of their respective receptors has been reported elsewhere. ${ }^{28} 29$

\section{Cell culture and stable clone production}

BOSC 23 retrovirus packaging cells were purchased from American Type Culture Collection (ATCC; Manassas, Virginia, USA). Firstly, we introduced the tet-Ang-2 vector into BNLHCC cells. A stable tet-Ang-2 expressing clone was obtained by the same method as described previously. ${ }^{30}$ Ang-2 expression levels in conditioned media were measured by ELISA (R\&D Systems, Minneapolis, Minnesota, USA). For further analysis, we used clones showing the highest and lowest Ang-2 in the absence or presence of tet $1 \mu \mathrm{g} / \mathrm{ml}$ (Sigma, St Louis, Missouri, USA), respectively (Ang-2-HCC). Next, we introduced Pcl-neo-VEGF into Ang-2-HCC cells with lipofectamin (Gibco, Rockville, Maryland, USA), selected with geneticin $(1 \mathrm{mg} / \mathrm{ml})$, as described previously (Ang-2-VEGFHCC). ${ }^{30}$ VEGF expression levels were also measured by ELISA (R\&D Systems). The Pcl-neo vector was also transfected into
A

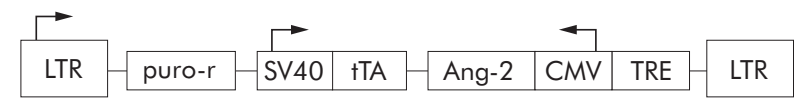

B

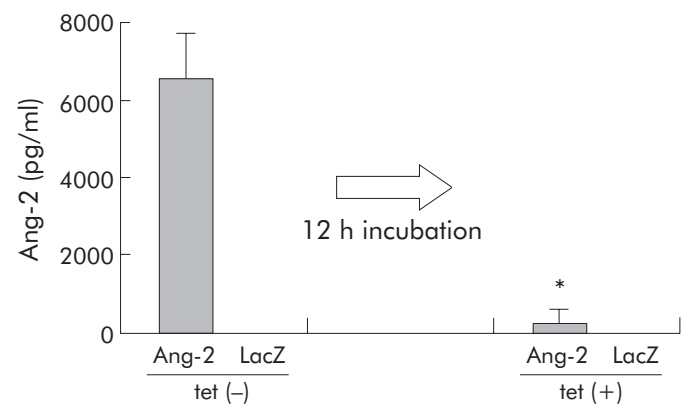

Figure 1 (A) Construction of the tetracycline (tet) regulated retrovirus mediated angiopoietin 2 (Ang-2) expression vector. The vector contains a tet controlled transcriptional activator (tTA) and a tet responsive element (TRE) in the opposite direction. Human Ang-2 was cloned into the multicloning site of the response unit. Transcriptional direction is indicated by an arrow. (B) Regulation of Ang-2 production by tet in vitro. Left: Human Ang-2 levels in 24 hour culture supernatant of BNLhepatocellular carcinoma (HCC) cells. LacZ-HCC cells did not produce any human Ang-2 whereas Ang-2-HCC cells secreted high levels of Ang-2. Right: tet $(1 \mu \mathrm{g} / \mathrm{ml})$ mediated effect on Ang-2 production by the same cells following a 12 hour incubation with tet in the culture medium. See methods section for a description of each group. Each bar represents the mean (SD) of three separate experiments, $n=6$ each. ${ }^{*} \mathrm{p}<0.01$, significantly difference compared with tet $(-)$.

Ang-2-HCC cells to avoid the non-specific effect of vector introduction. In the control group, the tet-lacZ and Pcl-neo vectors were introduced (lacZ- HCC).

\section{In vitro proliferation assay}

In vitro proliferation was determined by MTT assay, as previously described. ${ }^{31}$ Briefly, cell proliferation was quantified via conversion of tetrazolium, 3-(-4,5-diethylthiazoyl-2yl)-2,5-diphenyltetrazolium bromide (MTT) by cells cultured on 96 well plates. Absorbance with a $540 \mathrm{~nm}$ filter represents conversion to formazan, which is directly proportional to the number of living cells. Absorbance was read with an ELISA plate recorder ( $n=6$ per group). We performed the in vitro proliferation assay in the presence or absence of tet.

\section{Animal treatment}

In an allograft tumour growth experiment, $1 \times 10^{6}$ Ang-2HCC, Ang-2-VEGF-HCC, and lacZ-HCC cells were syngenetically transplanted into the flank of BALB/c mice (Japan SLC, Hamamatsu, Japan). Group l (G1), lacZ-HCC cell group, served as a control. Animals in group 2 (G2) were Ang-2-HCC transplanted mice. This group drank tet free normal drinking water to maintain Ang-2 overexpression. Animals in group 3 (G3) were Ang-2-VEGF-HCC transplanted mice with tet containing drinking water $(\mathrm{l} \mathrm{mg} / \mathrm{kg})$ to suppress Ang-2 expression. Mice in group $4(\mathrm{G} 4)$ also received transplanted Ang-2-VEGF-HCC cells, and drank tet free normal water throughout the experiment to maintain both Ang-2 and VEGF overexpression. Tumours were measured twice a week using callipers, and tumour volume was calculated as described previously. ${ }^{32}$ To confirm that the effect of these angiogenic factors on tumour growth was consistent, we performed the same animal experiments twice, independently $(\mathrm{n}=14$, the total in each experimental group). To examine whether Ang-2 gene expression was also tightly regulated by tet in vivo, five mice from G3 and G4 were killed 
three days after changing the drinking water. Subsequently, half of each tumour was snap frozen and the remainder was used for histological analysis. Another experiment was performed to examine the effects of R-1mAb and R-2mAb on VEGF or Ang-2-VEGF induced tumour development. $\mathrm{R}-\operatorname{lmAb}$ or $\mathrm{R}-2 \mathrm{mAb}$ was injected intraperitoneally twice a week at a dose of $800 \mu \mathrm{g} /$ mouse into VEGF overexpressing HCC cells (G5 and G6, respectively). In G7 and G8, R-1mAb and $\mathrm{R}-2 \mathrm{mAb}$ were also injected intraperitoneally into Ang-2VEGF overexpressing HCC cells, respectively. IgG was injected into VEGF-HCC, Ang-2-VEGF overexpressing HCC, and lacZ-HCC control groups, as described previously $(\mathrm{n}=10$ for each group). ${ }^{30}$

\section{Evaluation of mRNA expressions by real time polymerase chain reaction}

mRNA expression of Ang-1, Ang-2, VEGF, Tie-2, and $\alpha$ smooth muscle actin $(\alpha$-SMA) was evaluated by real time polymerase chain reaction (PCR), as described previously. ${ }^{30}{ }^{33}$ mRNA was extracted from the tumour of the animals in each experimental group $(\mathrm{n}=5)$. For cDNA synthesis, Taqman reverse transcription reagents were used as described in the manufacturer's manual of the ABI Prism 7700 Sequence Detection System (PE Applied Biosystems, Foster City, California, USA), which was used for real time PCR amplification following the Taqman Universal PCR Master Mix Protocol (PE Applied Biosystems). Relative quantitation of gene expression was performed as described in the manual, using glyceraldehyde-3-phosphate dehydrogenase as an internal control. The threshold cycle and standard curve method were used for calculating the relative amount of the target RNA, as described for PE. The following temperatures were used: hold at $50^{\circ} \mathrm{C}$ for two minutes, hold at $60^{\circ} \mathrm{C}$ for 30 minutes, hold at $94^{\circ} \mathrm{C}$ for five minutes, cycle 45 repeats at $94^{\circ} \mathrm{C}$ for one minute, at $55^{\circ} \mathrm{C}$ for one minute, and at $72^{\circ} \mathrm{C}$ for one minute. To prevent genomic DNA contamination, all RNA samples were subjected to DNase I digestion and checked by 40 cycles of PCR to confirm the absence of any amplified DNA.

\section{Immunohistochemistry}

For determination of in vivo angiogenesis, we used immunohistochemical detection of CD31, which is widely used as a marker of neovascularisation, in frozen sections of tumours. We used tumours of the same size to avoid necrotic effects of hypoxia. The length of the immunostained microvessel was assessed by light microscopy $(200 \times)$. Microvessel density (MVD) of the tumour was evaluated simultaneously by two independent observers, as described previously, without knowledge of the animal data. ${ }^{34}$ Briefly, after immunostaining with CD31 antibody, the tumour was first screened at low power $(\times 40)$ to identify areas of the highest MVD. MVD evaluation was performed in the five highest MVD areas at high power $\left(\times 200,0.74 \mathrm{~mm}^{2}\right.$ per field $)$. Microvessels that stained positively with anti-CD31 antibody were evaluated in five microscopic fields of the neovascularisation with the highest density. The mean value of the fields evaluated by each observer was considered as the MVD of the individual tumour. When counting, large vessels with a thick muscular wall or with a lumen greater than $50 \mu \mathrm{m}$ in diameter were excluded. Immunopositive vessels were evaluated with Adobe Photoshop and NIH image software, as described previously. ${ }^{30}$ Immunostaining of TUNEL positive apoptotic cells was performed as previously described using paraffin embedded sections. ${ }^{1433}$

\section{MMP-2 and MMP-9 expression in the tumour}

Tumour lysates which had equal protein concentrations were prepared as described previously. ${ }^{30}$ Expression levels of matrix metalloproteinases 2 (MMP-2) and 9 (MMP-9) were measured by an ELISA detection kit (Biotrak: Amersham Pharmacia Biotech, Tokyo, Japan) according to the manufacturer's instructions. This kit can measure both total and active forms of MMPs. In order to measure total MMP content, any bound MMP in its proform was activated using p-aminophenylmercuric acetate (APMA). The standards are proMMPs, which are activated in parallel for both types of samples. Active MMPs are detected without APMA treatment. We measured the active form of MMPs in each sample in triplicate $(\mathrm{n}=5)$.

\section{Statistical analysis}

To assess the statistical significance of intergroup differences in quantitative data, Bonferroni's multiple comparison test was used after one way ANOVA. This was followed by Barlett's test to determine the homology of variance.

\section{RESULTS}

\section{Regulation of transgenic Ang-2 and VEGF in vitro}

We first examined in vitro Ang-2 regulation in Ang-2-HCC cells. LacZ-HCC control cells did not show any evidence of
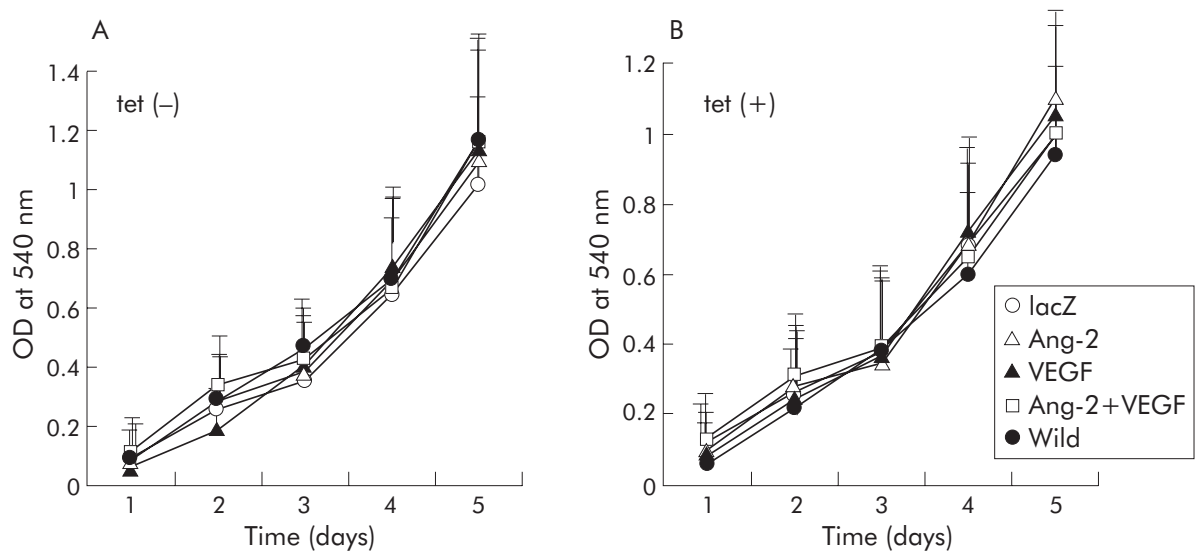

Figure 2 In vitro proliferation of lacZ-hepatocellular carcinoma (HCC), angiopoietin 2 (Ang-2)-HCC, vascular endothelial growth factor (VEGF)-HCC, and Ang-2-VEGF-HCC cells in culture in the absence (A) or presence (B) of tetracycline (tet). Cell proliferation was measured by the MTT assay after harvest from day 1 to day 5, as described in the methods section. There were no differences between the lacZ-HCC, Ang-2-HCC, VEGF-HCC, and Ang-2-VEGF-HCC cells, or in the presence or absence of tet. A description of each group is given in the methods section. Each bar represents the mean (SD) of three separate experiments. ( $n=6$ each). 


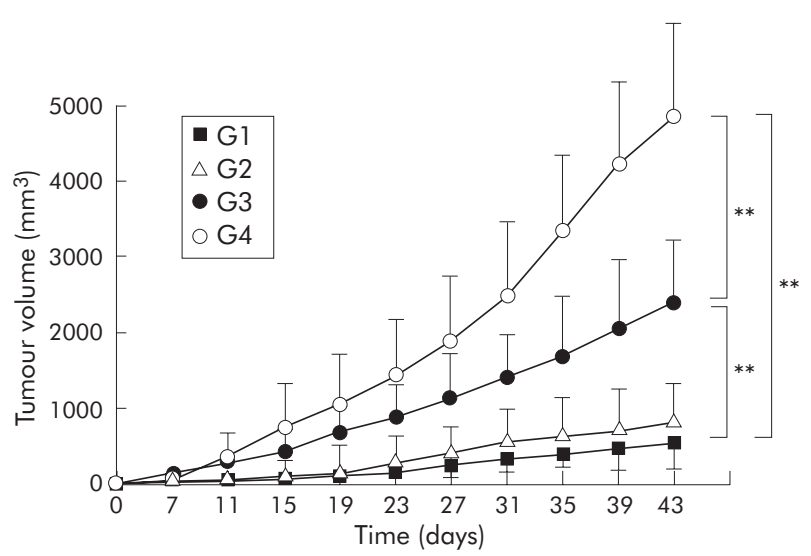

Figure 3 Effects of angiopoietin 2 (Ang-2) and vascular endothelial growth factor (VEGF) overexpression on BNL-hepatocellular carcinoma (HCC) growth. Group 3 (G3: VEGF-HCC) revealed a marked increase in tumour development $(p<0.01)$ compared with group 1 (G1: lacZ-HCC). On the other hand, tumour growth in group 2 (G2: Ang-2-HCC) was of a similar magnitude to that in G1. Group 4 (G4: Ang-2-VEGF-HCC) revealed a markedly increased level of tumour development, and this tumour growth stimulatory effect was much more than the additive effects of $G 2$ and $G 3$. A description of each group is given in the methods section. Each point represents the mean (SD) of 14 animals. ${ }^{* *} p<0.01$, significant differences between the indicated groups.

human Ang-2 production. In the absence of tet, marked secretion of Ang-2 was seen in the supernatant (6445.4 ( 1421.1$) \mathrm{pg} / \mathrm{ml}, \mathrm{n}=6)$. When tet $(1 \mu \mathrm{g} / \mathrm{ml})$ was added to the medium and incubated for an additional 12 hour period, Ang-2 protein level was markedly reduced (234.6 (44.6) $\mathrm{pg} / \mathrm{ml})(\mathrm{p}<0.01)$ (fig $\mathrm{l}$ ). In cell culture assays, the optimal concentration of VEGF to induce proliferation or survival response in endothelial cells is approximately $10 \mathrm{ng} / \mathrm{ml}$ whereas the optimal concentration of Ang-2 that evokes a response is more than $100 \mathrm{ng} / \mathrm{ml}$. Accordingly, 10-fold higher amounts of Ang-2 compared with VEGF were injected to examine the exogenous effects of both factors in vivo. ${ }^{21}$ In the current study, we also chose a stable VEGF expressing clone with this ratio, which expressed a level of $568.2(62.4) \mathrm{pg} / \mathrm{ml}$ $(\mathrm{n}=6)$ for Ang-2-VEGF-HCC cells. In the Ang-2-VEGF-HCC cells, Ang-2 regulation appeared to be the same as in Ang-2HCC cells, and in the presence or absence of tet, VEGF expression levels did not change (data not shown).

\section{Effects of Ang-2 and VEGF on proliferation of HCC cells in vitro}

In vitro proliferation rates for HCC cells from day 1 to day 5 after harvest were examined in the presence or absence of tet. As shown in fig 2, there were no differences between lacZHCC, Ang-2-HCC, VEGF-HCC, and Ang-2-VEGF-HCC cells in the presence or absence of tet, indicating that VEGF and Ang2 overexpression did not affect in vitro proliferation rates. We also performed an in vitro proliferation assay using the $\left[{ }^{3} \mathrm{H}\right]$ uptake method, and we obtained similar results (data not shown).

\section{Effects of Ang-2 and VEGF on HCC tumour growth, angiogenesis, and apoptosis}

We next examined the effects of Ang-2 and VEGF on tumour development in vivo. Ang-2-HCC and Ang-2-VEGF-HCC cells were injected into the flank of BALB/C mice. Similar to previous findings, ${ }^{13}$ the VEGF overexpression group (G3) revealed a marked increase in tumour development $(\mathrm{p}<0.01)$ compared with the lacZ-HCC control group (Gl). On the other hand, tumour growth in the Ang-2 overexpression

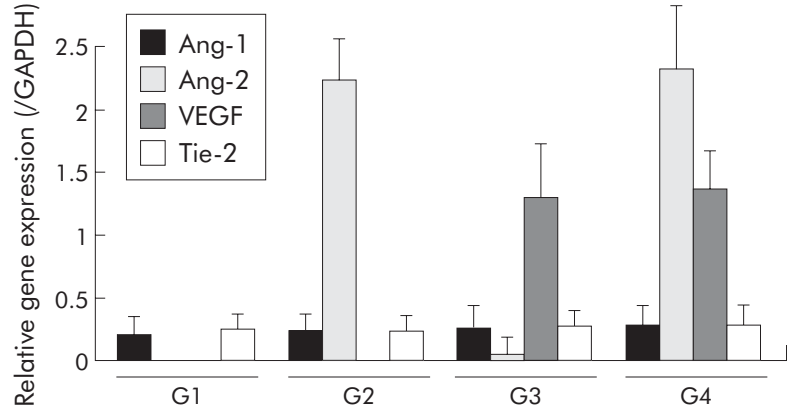

Figure 4 mRNA expressions of angiopoietin 1 (Ang-1), angiopoietin 2 (Ang-2), vascular endothelial growth factor (VEGF), and Tie-2 in the tumour. mRNA expression in the tumour in each experimental group was measured by real time polymerase chain reaction, as described in the methods section. Gene regulation of transgenic Ang-2 and VEGF expression in tumours was maintained at levels similar to those of cells in vitro. mRNA expression of endogenous Ang-1 and Tie- 2 constitutively expressed relatively low levels. G1, lacZ-hepatocellular carcinoma (HCC) group; G2, G3, Ang-2-HCC and VEGF-HCC groups, respectively; G4, Ang-2-VEGF-HCC group. A description of each group is shown in the methods section. Each bar represents the mean (SD) $(\mathrm{n}=5$ each).

group (G2) was of similar magnitude to that of G1. Combined expression of Ang-2 and VEGF (G4) revealed a markedly increased level of tumour development, and this tumour growth stimulatory effect was much more than the additive effect of the Ang-2 (G2) and VEGF (G3) groups (fig 3). As shown in fig 4, gene regulation of transgenic Ang-2 and VEGF expression in the tumours were maintained at levels similar to those of cells in vitro. We also examined mRNA expression of endogenous Ang- 1 and Tie- 2 and found that these genes constitutively expressed relatively low levels. Endogenous murine Ang-2 and VEGF expression levels were not altered by transgenic overexpression of the respective gene in the tumour, and tet did not have any effect on the healthy state (such as body weight) during the experiment (data not shown).

To determine whether the increased tumour growth rate was associated with Ang-2 and VEGF induced neovascularisation, we next examined the number of CD31 positive microvessels in the tumour. We found that VEGF overexpression (G3) caused a significant increase in CD31 immunopositive vessels of the tumour compared with the control group (Gl) whereas there was no significant difference between the Ang-2 overexpression group (G2) and Gl (fig 5). Similar to tumour growth, combined overexpression of Ang-2 and VEGF (G4) revealed a markedly increased level of CD31 positive vessels. The magnitude of CD31 positive vessel augmentation is likely to correspond to tumour development. As it has been demonstrated recently that image analysis was more reliable and objective than manual counting of microvessels, we employed computer assisted image analysis techniques, as described previously. ${ }^{35}$

Semiquantitative analysis of CD3 I positive vessels revealed that neovascularisation in tumours consisting of VEGF-HCC and Ang-2-VEGF-HCC cells showed a marked increase compared with the control group (fig 6A). In contrast, a marked decrease in apoptotic cells was found in the VEGF and Ang-2 plus VEGF overexpressing tumours compared with either the control or Ang-2 overexpressing tumours (fig 6B). It has been reported that the vessel maturation index, defined as the fraction of vessels associated with $\alpha$-SMA positive cells around EC, was significantly decreased in the Ang-2 overexpressing tumour. ${ }^{36}$ We therefore examined $\alpha$-SMA expression in the tumour. Similar to results for 

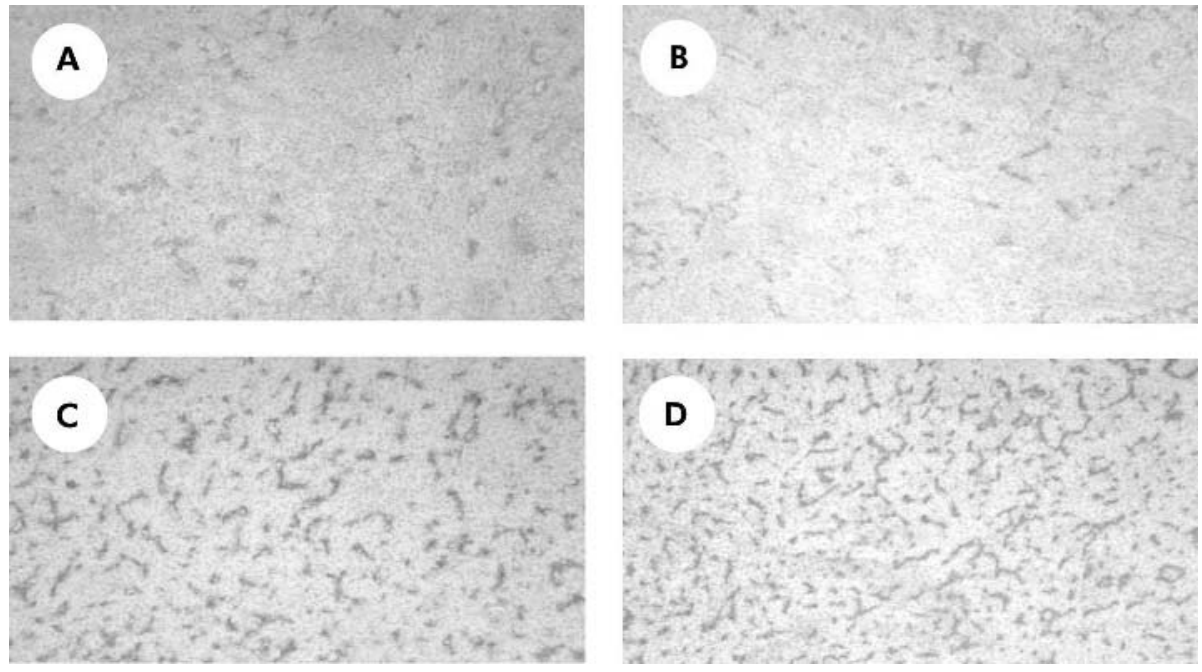

Figure 5 Effects of angiopoietin 2 (Ang-2) and vascular endothelial growth factor (VEGF) on CD31 expression in the tumours. Tumour vascularisation was visualised by immunostaining of the CD31 vascular endothelial adhesion protein. (A) LacZ-hepatocellular carcinoma (HCC) control group (G1). (B) Ang-2-HCC group (G2). (C) VEGF- HCC group (G3). (D) Ang-2-VEGF-HCC group (G4). G3 showed a significant increase in CD31

immunopositive vessels in tumour compared with controls (G1) whereas there was no significant difference between $G 2$ and $G 1$. Similar to tumour growth, G4 revealed a markedly increased level of CD31 positive vessels compared with G1 and G3. The magnitude of CD31 positive vessel augmentation is likely to correspond to tumour development. A description of each group is shown in the methods section. Original magnification $\times 200$.

apoptosis, intratumoral $\alpha$-SMA expression was significantly attenuated in Ang-2 plus VEGF overexpressing HCC (fig 6C).

We next examined the effects of R-1mAb and R-2mAb on Ang-2 and VEGF induced tumour growth in vivo. Similar to previous findings, ${ }^{15} \mathrm{R}-1 \mathrm{mAb}$ and $\mathrm{R}-2 \mathrm{mAb}$ treatment ( $\mathrm{G} 5$ and G6, respectively) significantly attenuated VEGF induced HCC tumour growth (G3). R-1mAb and R-2mAb (G7 and G8, respectively) also markedly suppressed Ang-2 plus VEGF overexpressing tumour (G4) (fig 7). Noteworthy was the finding that the suppressive effects of R-1mAb and R-2mAb against G4 were of a similar magnitude to the inhibitory effects against G3. Although both R-1mAb and R-2mAb significantly attenuated tumour development, the inhibitory effect of R-2mAb was more potent than that of R-1mAb. These results indicate that Ang-2 mediated proangiogenic and synergistic effects on tumour development are exerted only in the presence of VEGF, and depend on the VEGF signalling cascade, mainly via VEGFR-2.

\section{Effects of Ang-2 and VEGF on expression of MMP-2 and MMP-9 in the tumour}

The proangiogenic activity of Ang-2 is mediated, at least partly, via induction of MMPs. ${ }^{36}{ }^{37}$ To elucidate the possible mechanism of the Ang-2 mediated proangiogenic activity in the presence of VEGF, we examined the effects of Ang-2 and VEGF overexpression on active forms of MMP-2 and MMP-9 in the tumour. Similar to results for tumour growth, Ang-2 plus VEGF overexpression significantly upregulated the activities of MMP-2 and MMP-9 in the tumour (fig 8A and $8 \mathrm{~B}$, respectively). In contrast, MMP expression in the Ang-2 overexpression group (in the absence of VEGF) was similar to that in the control group. These results clearly indicate that
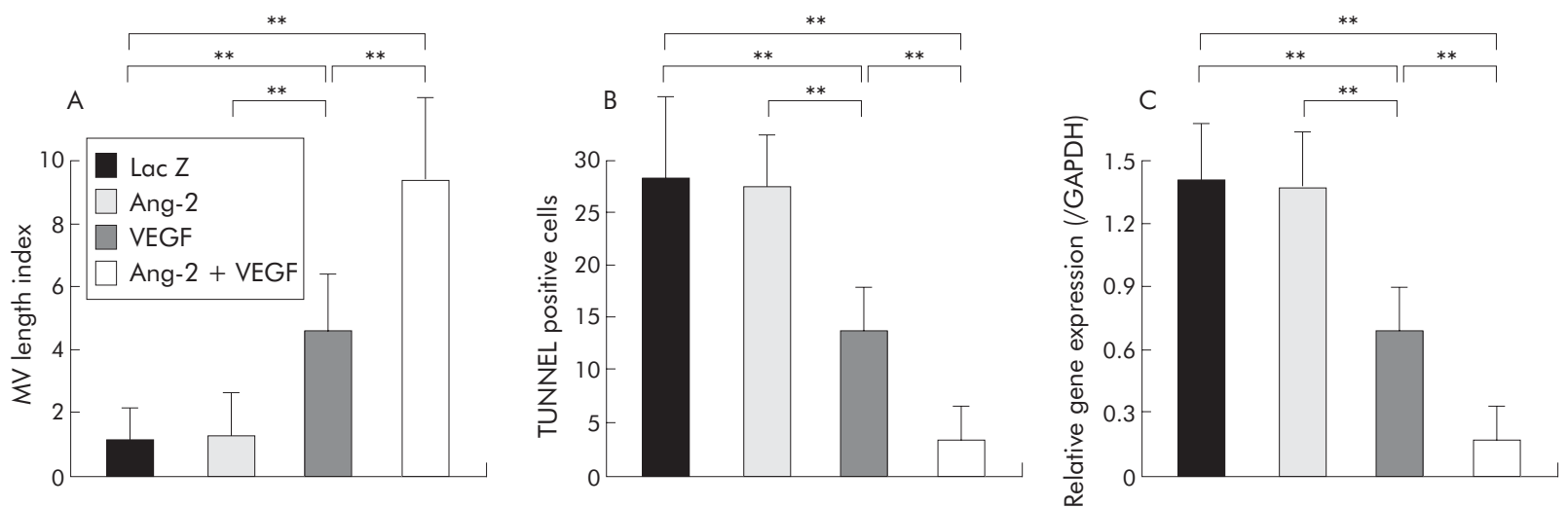

Figure 6 Semiquantitative analysis of CD31 immunopositive vessels (A), TUNEL immunopositive cells (B), and $\alpha$ smooth muscle actin ( $\alpha$-SMA) immunopositive cells (C). (A) Semiquantitative analysis of CD31 positive vessels revealed that neovascularisation in the tumours consisting of vascular endothelial growth factor (VEGF)-hepatocellular carcinoma (HCC) cells and angiopoietin 2 (Ang-2)-VEGF-HCC cells was markedly increased compared with the corresponding control group. The magnitude of CD31 positive vessel augmentation is likely to correspond to tumour development. (B, C) A marked decrease in TUNEL positive apoptotic cells was found in the VEGF and Ang-2 plus VEGF overexpressing tumours compared with either the control or Ang-2 overexpressing tumours. Inhibition of apoptosis correlated inversely with tumour development. Similar to the results of apoptosis, intratumoral $\alpha$-SMA expression was significantly attenuated in the Ang-2 plus VEGF overexpressing HCC. A description of each group and computer assisted image analysis are given in the methods section. Data are mean (SD) ( $n=5$ each). ${ }^{* *} p<0.01$, significant differences between the indicated groups. MV, microvessel. 


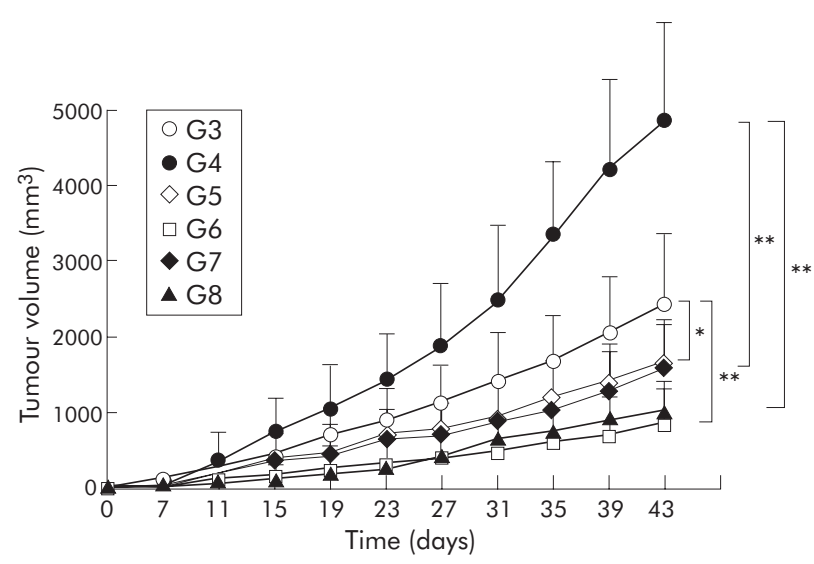

Figure 7 Effects of VEGFR-1 monoclonal antibody (mAb) and VEGFR-2 $\mathrm{mAb}$ on angiopoietin 2 (Ang-2) and vascular endothelial growth factor (VEGF) overexpression induced hepatocellular carcinoma (HCC) growth. R- $1 \mathrm{mAb}$ and $R-2 \mathrm{mAb}$ treatment (groups 5 and $6(\mathrm{G} 5, \mathrm{G} 6)$, respectively) significantly attenuated VEGF induced HCC tumour growth (group $3(G 3)$ ). R- $1 \mathrm{mAb}$ and $\mathrm{R}-2 \mathrm{mAb}$ (groups 7 and $8(G 7, G 8)$ ), respectively) also markedly suppressed Ang-2 plus VEGF overexpressing tumour (G4). Although both R-1mAb and R-2mAb significantly attenuated tumour development, the inhibitory effect of $R-2 m A b$ was more potent than that of $R-1 \mathrm{~mA} b$. A description of each group is given in the methods section. Data are mean (SD) ( $n=10$ each). ${ }^{*} p<0.05$, ${ }^{* *} p<0.01$, significant differences between the indicated groups.

Ang-2 mediated MMP-2 and MMP-9 induction is exclusively dependent on VEGF expression in the tumour.

We next examined whether inhibition of VEGF abrogated the effect of transgenic Ang-2 and VEGF on MMP-2 and MMP-9 levels in the tumour, using R-1mAb and R-2mAb. As shown in fig 9, R-1mAb and R-2mAb significantly suppressed both MMP-2 and MMP-9 activities. These inhibitory effects mostly corresponded to tumour growth suppression.

\section{DISCUSSION}

HCC is recognised as a distinctively hypervascular tumour in clinical practice. ${ }^{1}$ Among the proangiogenic factors, VEGF is the most intriguing with regard to the angiogenic process. ${ }^{7}$ In HCC it has been reported that VEGF was highly expressed in tumour lesions compared with the adjacent non-cancerous lesions, ${ }^{38}$ and overexpression of VEGF significantly enhanced HCC tumour development and angiogenesis. The degree of tumour enlargement corresponded to the level of VEGF expression. ${ }^{13}$ Despite its requisite role in tumour vascular formation, VEGF must work in concert with other factors. We previously reported that VEGF and basic fibroblast growth factor (bFGF) showed a synergistic effect on HCC development and angiogenesis. We also found that bFGF induced proangiogenic activity was partly mediated by induction of VEGF through VEGFR-2 activation. ${ }^{30}$ Alternatively, Ang-2 seems to be one of most important partners. In human HCC, high expression of Ang-2 compared with adjacent noncancerous tissues has been reported. ${ }^{18} 193940$ The action of Ang-2 is still controversial but recent experiments have shown that in some circumstances this factor can promote angiogenesis, including HCC tumour growth. ${ }^{16}{ }^{18}$ Ang-2 facilitates the angiogenic response in the presence of VEGF whereas it causes vessel regression without VEGF. ${ }^{20}{ }^{21}$ It has also been reported that Ang-2 displayed VEGF dependent modulation of the capillary structure and EC survival in a corneal assay. ${ }^{21}$

In the current study, we have shown that Ang-2 synergistically augmented HCC development and angiogenesis only in the presence of VEGF. Apart from being an angiogenic factor, VEGF is also known as a survival factor for the newly formed tumour EC. Without VEGF, vessel regression via apoptosis of EC rapidly occurs. ${ }^{41}$ Ang-2 at high concentrations has also been shown to act as an antiapoptotic factor through the PI3 kinase/Akt signalling pathway in vitro. $^{42}$ Tumour growth enhancement by proangiogenic factors was associated with a marked decrease in apoptosis in the tumour whereas it did not significantly affect tumour cell proliferation itself. ${ }^{5}$ In vitro, we also found that neither Ang-2 nor VEGF stimulated HCC cell proliferation. On the other hand, a marked decrease in apoptotic cells was observed in the Ang-2 plus VEGF overexpressing tumour compared with either the control or Ang-2 overexpressing tumour. These results suggest that an antiapoptotic effect of both molecules is involved in the synergistic effect on HCC development and angiogenesis. We also examined the synergistic effect of Ang-2 and VEGF using another clone, which expressed a different level of VEGF (274.2 (31.3) $\mathrm{pg} / \mathrm{ml})$. We found that the synergistic effect of tumour

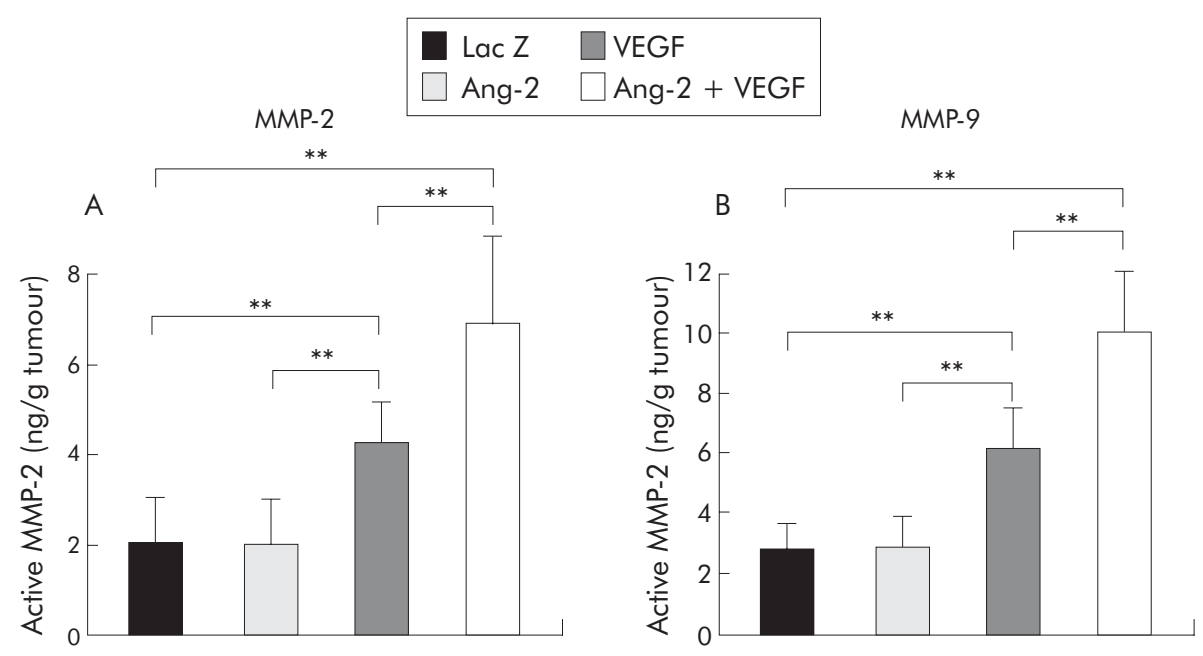

Figure 8 Effects of angiopoietin 2 (Ang-2) and vascular endothelial growth factor (VEGF) on active forms of matrix metalloproteinase (MMP)-2 (A) and MMP-9 (B) in the tumour. The activities of MMP-2 and MMP-9 in the tumour of VEGF-hepatocellular carcinoma (HCC) and Ang-2-VEGF-HCC were significantly increased compared to those of lacZ-HCC. On the other hand, MMP expression in the Ang-2-HCC group (in the absence of VEGF) was similar to that in the control group. A description of each group is given in the methods section. Data are mean (SD) ( $n=5$ each). ${ }^{* *} p<0.01$, significant differences between the indicated groups. 

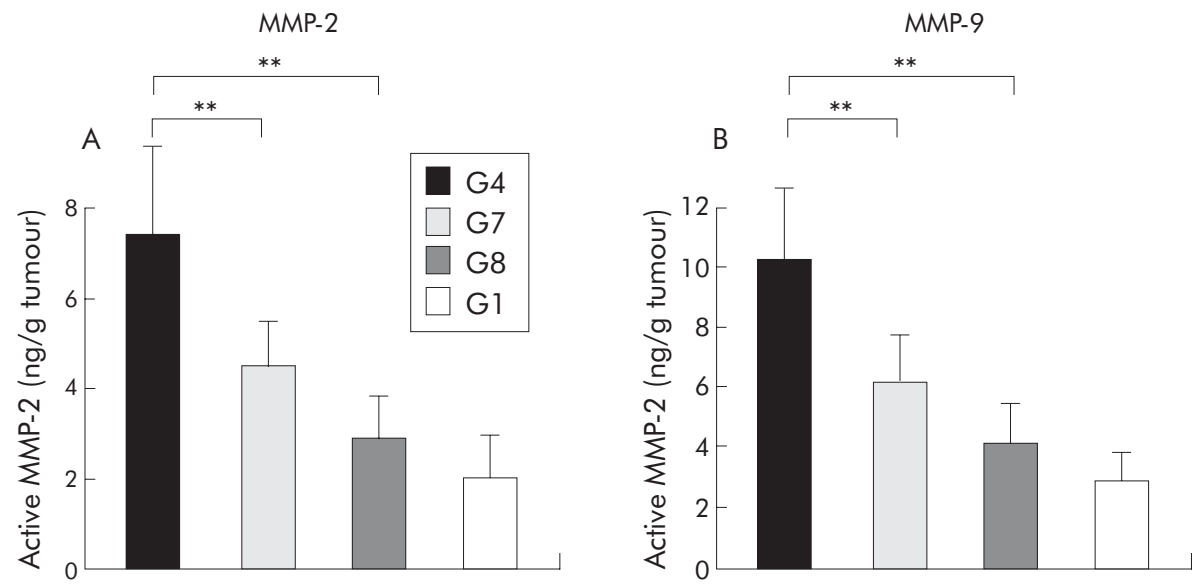

Figure 9 Effects of $R-1$ monoclonal antibody (mAb) and $R-2 m A b$ on active forms of matrix metalloproteinase (MMP)-2 (A) and MMP-9 (B) in the angiopoietin 2 (Ang-2) and vascular endothelial growth factor (VEGF) overexpression induced hepatocellular carcinoma (HCC) growth. R-1 mAb and R-2mAb (groups 7 and $8(G 7, G 8$ ), respectively) markedly suppressed the activity of both MMP-2 and MMP-9 in the Ang-2 plus VEGF overexpressing tumour (group $4(\mathrm{G} 4)$ ). The inhibitory effects of R-1mAb and R-2mAb on MMP activity mostly correlated with suppression of tumour growth. A description of each group is given in the methods section. Data are mean (SD) $\left(n=5\right.$ each). ${ }^{* *} p<0.01$, significant differences between the indicated groups.

development and angiogenesis seemed to be dependent on the transgenic VEGF level in the tumour (data not shown).

Previous reports have suggested that MMP upregulation plays an important role in Ang-2 mediated proangiogenic activity. $^{36}{ }^{37}$ Among the MMPs, MMP-2 and MMP-9 are reportedly overexpressed in HCC. ${ }^{43}{ }^{44}$ MMP-9 was upregulated by Ang-2 in the presence of VEGF whereas Ang-2 alone did not show such an effect in EC in vitro. ${ }^{36}$ Moreover, MMP-9 has been shown to be a trigger of the angiogenic switch during carcinogenesis in the transgenic mouse model, and a synthetic inhibitor of VEGF signalling impaired this angiogenic switch and tumour growth. ${ }^{45}$ Similarly, we observed that Ang-2 significantly stimulated MMP-9 expression only in the presence of VEGF in the HCC tumour. In addition to MMP-9, another report has shown that MMP-2 activation by Ang-2 played a critical role in inducing glioma cell infiltration. ${ }^{37}$ We also observed significant upregulation of MMP-2 in the Ang-2 plus VEGF overexpressing tumour. Taken together, induction of MMP-2 and MMP-9 by Ang-2 plus VEGF may also contribute to HCC development and angiogenesis.

VEGF was originally identified as a vascular permeability factor which had a potent ability to permeabilise capillaries to a level 50000 -fold higher than histamine. ${ }^{7}$ It induces extravasation of plasma proteins, such as fibrinogen, which when deposited in the extracellular matrix may serve as a foundation for the formation of new capillaries. Ang-1 has been shown to reduce the vascular permeability induced by VEGF. ${ }^{46}$ As Ang-2 is the natural antagonist to Ang-l, continuous overexpression of Ang-2 would facilitate the vascular permeability of VEGF in the tumour. Ang-2 also blocks the stabilisation effect of Ang-1 in the presence of VEGF. ${ }^{8}$ In the current study, we observed that $\alpha$-SMA expression was markedly attenuated in the Ang-2 plus VEGF overexpressing HCC tumour. These results suggest that tumour vessel Tie- 2 receptor blockade by continuous Ang-2 overexpression in the presence of VEGF would lead to failure of maturation of tumour vessels, thus contributing to HCC development and angiogenesis.

In HCC it has been suggested that the interaction between VEGF and Ang-2 may play an important role in tumour angiogenesis. ${ }^{40}$ There was a strong correlation between expressions of VEGF and Ang-2 in human HCC tissues, and this expression strongly correlated with intratumoral microvessel density. A synergistic effect of Ang-2 and VEGF on proliferation of EC in vitro has been reported.$^{47}$ It has been shown that VEGF upregulated the transcription of Ang-2, which is mediated via VEGFR-2 in EC. ${ }^{48}$ Although we did not examine whether or not VEGF directly affected Ang-2 expression in the tumour, we observed that tumour augmentation by Ang-2 plus VEGF was mediated, at least in part, via VEGFR-2 in the tumour. It has been reported that MEKl/2 and Akt are involved in the downstream signal of VEGFR-2-mediated VEGF bioactivities. ${ }^{7}$ We observed that phosphorylation of $\mathrm{MEKl} / 2$ and Akt seemed to correlate mostly with tumour growth augmentation by Ang-2 and VEGF (data not shown). It is possible that VEGFR-2 activation and downstream signals, such as MEKl/2 and Akt, are involved in the synergistic effect of Ang-2 and VEGF. The exact molecular mechanism that explains how this direct interaction between Ang-2 and VEGF contributes to the synergistic effect on HCC development and angiogenesis should be elucidated in the future.

In conclusion, we have revealed that Ang- 2 and VEGF have a synergistic effect on HCC development and angiogenesis by a combination of the Retro-Tet system and a conventional gene expression system. The proangiogenic and tumour growth stimulating effects of Ang-2 were achieved only in the presence of VEGF. These results suggest that although Ang-2 is an important factor, VEGF is a more prerequisite central key molecule in HCC development and angiogenesis.

\section{Authors' affiliations}

H Yoshiji, R Noguchi, J Yoshii, Y Ikenaka, K Yanase, T Namisaki, M Kitade, M Uemura, H Fukui, Third Department of Internal Medicine, Nara Medical University, Kashihara, Nara, Japan

S Kuriyama, T Masaki, Third Department of Internal Medicine, Kagawa University School of Medicine, Kagawa, Japan

Conflict of interest: None declared.

\section{REFERENCES}

1 Befeler AS, Di Bisceglie AM. Hepatocellular carcinoma: diagnosis and treatment. Gastroenterology 2002;122:1609-19.

2 Schafer DF, Sorrell MF. Hepatocellular carcinoma. Lancet 1999;353:1253-7.

3 Nakashima O, Sugihara S, Kage M, et al. Pathomorphologic characteristics of small hepatocellular carcinoma: a special reference to small hepatocellular carcinoma with indistinct margins. Hepatology 1995;22:101-5. 
4 Sakamoto $M$, Ino $Y$, Fujii T, et al. Phenotype changes in tumor vessels associated with the progression of hepatocellular carcinoma. Jpn J Clin Oncol 1993;23:98-104.

5 Kerbel RS. Tumor angiogenesis: past, present and the near future. Carcinogenesis 2000;21:505-15.

6 Carmeliet P. Angiogenesis in health and disease. Nat Med 2003;9:653-60.

7 Ferrara N, Gerber HP, LeCouter J. The biology of VEGF and its receptors. Nat Med 2003;9:669-76.

8 Loughna S, Sato TN. Angiopoietin and Tie signaling pathways in vascular development. Matrix Biol 2001;20:319-25.

9 Shibuya M. Structure and function of VEGF/VEGF-receptor system involved in angiogenesis. Cell Struct Funct 2001;26:25-35.

10 Karkkainen MJ, Petrova TV. Vascular endothelial growth factor receptors in the regulation of angiogenesis and lymphangiogenesis. Oncogene 2000;19:5598-605

11 Mise M, Arii S, Higashitsuji $\mathrm{H}$, et al. Clinical significance of vascular endothelial growth factor and basic fibroblast growth factor gene expression in liver tumor. Hepatology 1996;23:455-64.

12 Yamaguchi R, Yano H, Nakashima $Y$, et al. Expression and localization of vascular endothelial growth factor receptors in human hepatocellular carcinoma and non-HCC tissues. Oncol Rep 2000;7:725-9.

13 Yoshiji H, Kuriyama S, Yoshii J, et al. Vascular endothelial growth factor tightly regulates in vivo development of murine hepatocellular carcinoma cells. Hepatology 1998;28:1489-96.

14 Yoshiji H, Kuriyama S, Hicklin DJ, et al. KDR/Flk-1 is a major regulator of vascular endothelial growth factor-induced tumor development and angiogenesis in murine hepatocellular carcinoma cells. Hepatology 1999:30:1179-86.

15 Yoshiii H, Kuriyama S, Yoshii J, et al. Involvement of the vascular endothelial growth factor receptor-1 in murine hepatocellular carcinoma development. J Hepatol 2004;41:97-103.

16 Tait CR, Jones PF. Angiopoietins in tumors: the angiogenic switch. J Pathol 2004;204:1-10.

17 Yancopoulos GD, Davis S, Gale NW, et al. Vascular-specific growth factors and blood vessel formation. Nature 2000;407:242-8.

18 Tanaka S, Mori M, Sakamoto Y, et al. Biologic significance of angiopoietin-2 expression in human hepatocellular carcinoma. J Clin Invest 1999:103:341-5

19 Mitsuhashi N, Shimizu H, Ohtsuka M, et al. Angiopoietins and Tie-2 expression in angiogenesis and proliferation of human hepatocellular carcinoma. Hepatology 2003;37:1 105-13.

20 Asahara T, Chen D, Takahashi T, et al. Tie2 receptor ligands, angiopoietin-1 and angiopoietin-2, modulate VEGF-induced postnatal neovascularization. Circ Res 1998;83:233-40.

21 Lobov IB, Brooks PC, Lang RA. Angiopoietin-2 displays VEGF-dependent modulation of capillary structure and endothelial cell survival in vivo. Proc Natl Acad Sci U S A 2002;99:11205-10.

22 Visconti RP, Richardson CD, Sato TN. Orchestration of angiogenesis and arteriovenous contribution by angiopoietins and vascular endothelial growth factor (VEGF). Proc Natl Acad Sci U S A 2002;99:8219-24.

23 St Croix B, Rago C, Velculescu V, et al. Genes expressed in human tumor endothelium. Science 2000;289:1197-202.

24 Gossen $\mathrm{M}$, Bujard $\mathrm{H}$. Tight control of gene expression in mammalian cells by tetracycline-responsive promoters. Proc Natl Acad Sci U S A 1992;89:5547-51.

25 Lyden D, Hattori K, Dias S, et al. Impaired recruitment of bone-marrowderived endothelial and hematopoietic precursor cells blocks tumor angiogenesis and growth. Nat Med 2001;7:1194-201.

26 Hattori K, Heissig B, Wu Y, et al. Placental growth factor reconstitutes hematopoiesis by recruiting VEGFR1(+) stem cells from bone-marrow microenvironment. Nat Med 2002;8:841-9.

27 Yoshiji H, Kuriyama S, Hicklin DJ, et al. The vascular endothelial growth factor receptor KDR/Flk- 1 is a major regulator of malignant ascites formation in the mouse hepatocellular carcinoma model. Hepatology 2001;33:841-7.
28 Luttun A, Tiwa M, Moons L, et al. Revascularization of ischemic tissues by PIGF treatment, and inhibition of tumor angiogenesis, arthritis and atherosclerosis by anti-Flt1. Nat Med 2002;8:831-40.

29 Prewett M, Huber J, Li Y, et al. Antivascular endothelial growth factor receptor (fetal liver kinase 1) monoclonal antibody inhibits tumor angiogenesis and growth of several mouse and human tumors. Cancer Res 1999;59:5209-18.

30 Yoshiii H, Kuriyama S, Yoshii J, et al. Synergistic effect of basic fibroblast growth factor and vascular endothelial growth factor in murine hepatocellular carcinoma. Hepatology 2002;35:834-42.

31 Yoshiji H, Kuriyama S, Kawata M, et al. The angiotensin-l-converting enzyme inhibitor perindopril suppresses tumor growth and angiogenesis: possible role of the vascular endothelial growth factor. Clin Cancer Res 2001;7:1073-8.

32 Yoshiji H, Harris SR, Thorgeirsson UP. Vascular endothelial growth factor is essential for initial but not continued in vivo growth of human breast carcinoma cells. Cancer Res 1997;57:3924-8.

33 Yoshiji H, Kuriyama S, Yoshii J, et al. Angiotensin-II type 1 receptor interaction is a major regulator for liver fibrosis development in rats. Hepatology 2001;34:745-50.

34 Weidner N, Semple JP, Welch WR, et al. Tumor angiogenesis and metastasis-correlation in invasive breast carcinoma. N Engl J Med 1991;324:1-8.

35 Tanigawa N, Lu C, Mitsui T, et al. Quantitation of sinusoid-like vessels in hepatocellular carcinoma: its clinical and prognostic significance. Hepatology 1997;26:1216-23.

36 Etoh T, Inove H, Tanaka S, et al. Angiopoietin-2 is related to tumor angiogenesis in gastric carcinoma: possible in vivo regulation via induction of proteases. Cancer Res 2001;61:2145-53.

37 Hu B, Guo P, Fang Q, et al. Angiopoietin-2 induces human glioma invasion through the activation of matrix metalloprotease-2. Proc Natl Acad Sci U S A 2003; 100:8904-9.

38 Yoshiji H, Kuriyama S, Fukui H. Angiotensin-l-converting enzyme inhibitors may be an alternative anti-angiogenic strategy in the treatment of liver fibrosis and hepatocellular carcinoma. Possible role of vascular endothelial growth factor. Tumour Biol 2002;23:348-56.

39 Torimura $\mathrm{T}$, Ueno $\mathrm{T}$, $\mathrm{Kin} \mathrm{M}$, et al. Overexpression of angiopoietin-1 and angiopoietin-2 in hepatocellular carcinoma. J Hepatol 2004;40:799-807.

40 Moon WS, Rhyu KH, Kang MJ, et al. Overexpression of VEGF and angiopoietin 2: a key to high vascularity of hepatocellular carcinoma? Mod Pathol 2003;16:552-7.

41 Bruns CJ, Liu W, Davis DW, et al. Vascular endothelial growth factor is an in vivo survival factor for tumor endothelium in a murine model of colorectal carcinoma liver metastases. Cancer 2000;89:488-99.

$42 \mathrm{Kim} \mathrm{I}, \mathrm{Kim} \mathrm{JH}$, Moon SO, et al. Angiopoietin-2 at high concentration can enhance endothelial cell survival through the phosphatidylinositol 3'-kinase/ Akt signal transduction pathway. Oncogene 2000;19:4549-52.

43 Maatta M, Soini Y, Liakka A, et al. Differential expression of matrix metalloproteinase (MMP)-2, MMP-9, and membrane type 1-MMP in hepatocellular and pancreatic adenocarcinoma: implications for tumor progression and clinical prognosis. Clin Cancer Res 2000;6:2726-34.

44 Arii S, Mise M, Harada T, et al. Overexpression of matrix metalloproteinase 9 gene in hepatocellular carcinoma with invasive potential. Hepatology 1996:24:316-22.

45 Bergers G, Brekken R, McMahon G, et al. Matrix metalloproteinase-9 triggers the angiogenic switch during carcinogenesis. Nat Cell Biol 2000;2:737-44.

46 Thurston G. Complementary actions of VEGF and angiopoietin-1 on blood vessel growth and leakage. J Anat 2002;200:575-80.

47 Huang XL, Takakura N, Suda T. In vitro effects of angiopoietins and VEGF on hematopoietic and endothelial cells. Biochem Biophys Res Commun 1999;264:133-8

48 Zhang L, Yang N, Park JW, et al. Tumor-derived vascular endothelial growth factor up-regulates angiopoietin-2 in host endothelium and destabilizes host vasculature, supporting angiogenesis in ovarian cancer. Cancer Res 2003;63:3403-12. 